\title{
Mechanisms of action of regulatory lymphocytes and a possibility to use them in the treatment of autoimmune diseases and $\mathrm{GvH}$ reactions
}

\author{
Buc M \\ Institute of Immunology, Comenius University Faculty of Medicine, Bratislava, Slovakia. \\ milan.buc@fmed.uniba.sk
}

\begin{abstract}
Basic characteristic of the immune system is its ability to distinguish self-molecules, cells, tissues and organs from not self, to tolerate self and dispose of not self. Immunosuppressive mechanisms, especially those mediated by regulatory lymphocytes, play a paramount role in the tolerance mechanisms. When there is an abnormal quantity and/or quality of regulatory cells, various autoimmune diseases are induced, e.g. SLE, RA, T1D, IBD, MS, and others.

In recent years, a great progress was achieved in the field how to profit from immunosuppressive properties of $\mathrm{T}$ regulatory cells (Treg) in the treatment of patients suffering from autoimmune disorders or transplantation rejections. Nowadays, there are possibilities to up-regulate the function of patient's Tregs or supplement their low numbers. We can up-regulate the function of Treg cells in an affected organism by treatment by low dosage of IL-2 or to treat patients by in vitro expanded Treg cells themselves. Induced Treg $_{\mathrm{s}}$ are, however, polyspecific, therefore they have been preferentially used for the treatment graft versus host reactions and some autoimmune disorders only. For those autoimmune diseases, where specific autoantigens are known, Treg cells equipped by antigen-specific chimeric T cell receptor (CARs) were introduced for their treatment (Tab. 1, Fig. 2, Ref. 47). Text in PDF www.elis.sk. KEY WORDS: AIRE, autoimmune diseases, CAR, cytokines, iNKT cells, regulatory B and T cells.
\end{abstract}

\section{Introduction}

Induction and maintenance of immunological tolerance to selfantigens and innocuous environmental antigens represents a basic principle of prevention of autoimmune processes and chronic inflammation. Immunosuppressive mechanisms are also very important in keeping the extent of inflammation on an appropriate level. Therefore, in healthy persons, fine balance is created between a pro-inflammatory response, which is needed to overcome infection and an immunosuppressive response, which prevents chronic inflammation and tissue damage. The immune system has developed various mechanisms preventing its reaction to autoantigens, either by physical elimination of autoreactive lymphocytes in the thymus or by hindering their activation in the periphery. This latter function is mediated mainly by regulatory $\mathrm{T}$ and $\mathrm{B}$ cells.

Regulatory T cells arise both in the thymus (natural Treg cells) and in the periphery (peripheral Treg cells) as the consequence of exposure to antigens. Natural regulatory T cells $\left(\mathrm{nTreg}_{\mathrm{s}}\right)$ down-

Institute of Immunology, Comenius University, School of Medicine, Bratislava, Slovakia

Address for correspondence: M. Buc, MD, DSc, Institute of Immunology, Comenius University, School of Medicine, Odborarske nam 14, SK81372 Bratislava, Slovakia.

Phone: +421290119398 regulate activities of T cells (so $\alpha \beta$ T cells as well as $\gamma \delta$ T cells), B cells, NK and iNKT cells, dendritic cells and macrophages. nTreg cells differentiate in the thymus as an independent population of naive $\mathrm{CD}^{+} \alpha \beta$ T cells. Treg cells differentiation requires activation of a specific gene FOXP3 (forkhead box P3; Xp11.23-q13.3) coding for the transcription factor FOXP3; it is responsible for activation or repression of more than 1,400 genes (1-3). However, it itself appears in the 2nd phase of Treg cells development only. The 1st differential signal is provided by $\mathrm{T}$ cells receptor recognizing self-molecules in membranes of epithelial and dendritic cells of the medulla of the thymus. It results in the expression of CD25 (alpha chain of high-affinity IL-2 receptor) and characteristic membrane molecules GITR, OX40, TNFR2. Subsequent activation of developing Treg cells by IL-2 results in FOXP3 expression (4, 5). A mutation of FOXP3 (more than 75 different mutations were described till now) proves its great biological significance - a fatal disease (IPEX - immune dysregulation, polyendocrinopathy, and enteropathy) will develop $(6,7)$.

Treg cells represent 5-10\% of CD4 $4^{+} \mathrm{CD} 8-$ thymocytes and in the periphery, approximately $10 \%$ of $\mathrm{CD}^{+} \mathrm{T}$ cells. They are long lived cells characterized by multiple molecules, esp. CD4 and CD25. However, they are not specific for Treg cells only. CD4 is expressed by all Thelper cells (Th) and CD25 shows an increased expression on large proportion of activated effector T cells. Later, it was found that nTreg cells expressed low quantity of CD127 
(alpha chain of IL-7 receptor) (8); however, it also cannot be a specific feature of Treg cells because activated $\mathrm{CD} 4^{+} \mathrm{Th}$ cells decrease CD127 quantity in their membranes.

nTreg cells are naturally anergic (i.e. they do not proliferate and do not synthesize IL-2 after in vitro stimulation by anti-CD3 monoclonal antibodies, phytohaemagglutinin or allogenic cells). However, they significantly inhibit induction of the immune response. A variety of molecules are found to be involved in Treg-mediated suppressive activity, including cytotoxic T-lymphocyte associated protein 4 (CTLA-4), IL-10, TGF- $\beta$, IL-35, glucocorticoid-induced TNF receptor (GITR), lymphocyte-activation gene 3 (LAG3), granzyme B, adenosine, cyclic AMP (cAMP), and trogocytosis (9-11).

The results of research in recent years showed that the thymus is continually releasing the autoreactive $T$ cells to the periphery to greater extent than we have thought so far and that their activation is practically permanent. Their differentiation into fully functional effector cells is blocked by nTreg lymphocytes. Also, migrating dendritic cells consistently deliver autoantigens from tissues and organs to the lymph nodes, which in turn recognize autoreactive $\mathrm{T}$ cells that, upon activation, could trigger autoimmune processes. They are, however, prevented by nTreg cells, which also recognize the presented autoantigens. In addition, IL-2, which activates effector T cells production, expands them and creates a microenvironment that prevents the induction of autoimmune processes. This interaction between migratory DCs and effector and regulatory lymphocytes proceeds at the periphery in paracortical regions of lymph nodes, in clusters separated from each other $(12,13)$. This finding once again confirmed our prior knowledge that IL-2 is not the major cytokine needed to develop an effector immune response as originally thought, but that it is a cytokine that ensures the existence and activity of nTreg cells.

Regulatory $\mathrm{T}$ cells can also appear at the periphery. These are peripheral regulatory $\mathrm{T}$ cells ( $\mathrm{p}$ Treg) that differentiate from naive $\mathrm{CD}^{+} \mathrm{T}$ cells during an ongoing immune response. They are induced mainly in mucosal areas; we know the best of these events in the intestine. If naive $\mathrm{CD}^{+} \mathrm{T}$ cells are stimulated by TGF- $\beta$, IL-2 and retinoic acid, which dendritic cells produce by processing vitamin A, they differentiate into pTreg cells (14). Most recently, it has been found that they can appear under the influence of IL-33, which is constitutively expressed in epithelial cells of the intestine and, upon their damage, it is released into the environment and acts as an alarmin (15). Upon binding to its receptor, ST2, in membranes of pTregs together with the signaling triggered by TGF- $\beta$, IL-33 promotes activation of transcription factor GATA3, a known transcriptional regulator of the FOXP3 gene. In addition, IL-33 also enhances its own receptor synthesis, increasing the persistence of pTreg cells in the given microenvironment. Strikingly, IL-23, the key pro-inflammatory cytokine in the pathogenesis of inflammatory bowel disease (IBD), restrained Treg responses through inhibition of IL-33 responsiveness. These results suggest that the balance between IL-33 and IL-23 may be the key controller of intestinal immune responses (16).

Compared to nTreg cells, pTreg cells are less stable and in the inflammatory environment they may lose the ability to express FOXP3 and vice versa, they synthesize pro-inflammatory cytokines such as IFN- $\gamma$ and IL-17. This lack of stability can be explained by the methylation state of the CNS2-region (conserved non-coding DNA sequence) in the promoter region of the FOXP3 gene. This region is stably hypomethylated in nTreg cells even in the inflammatory environment, while it is incompletely demethylated in iTreg cells $(17,18)$ (methylation of genes results in their decreased transcription).

In addition to the above mentioned, there is another population of immunosuppressive cells, referred to as Tr1 cells. They are induced by the simultaneous action of IL-27 and TGF- $\beta$ on naive $\mathrm{T}$ helper cells and produce mainly IL-10. In addition, they also inhibit the immune response by killing antigen presenting cells by a perforin-granzyme mechanism. In addition to CD4, CD49b and LAG-3 (CD223) characterize their specific features. Tr1 cells are FOXP3-negative, instead they express multiple transcription factors typical of other T cells subsets, e.g. c-MAF, AHR (aryl hydrocarbon receptor) and others (Tab. 1) (19, 20).

The main biological function of Treg cells, as described above, is to maintain immune and inflammatory processes at physiological boundaries. Their lack of, or malfunction, inevitably results in the development of autoimmune diseases. Functional impairment has been demonstrated in the development of type 1 diabetes mellitus (T1D) (21), rheumatoid arthritis $(22,23)$ myasthenia gravis (24), multiple sclerosis $(25,26)$ and other diseases $(27)$. The reduction of their numbers was confirmed at Crohn' disease and ulcerative colitis (28), Graves' disease (29) and hepatitis C virus-induced vasculitis (30). In systemic lupus erythematosus (SLE), the persistent proinflammatory cytokine environment prevents Treg lymphocytes from enforcing their immunosuppressive action (31).

Decreased numbers of nTreg cells are also observed in allergic patients, resulting in a reduced control of Th2-lymphocyte activation (3). Here too, the hygienic hypothesis is confirmed - insufficient activation of Th1 cells also causes low IL-2 production and hence appearance of nTreg cells.

IL-2 has a decisive influence on the formation and maintenance of nTreg cells activity. This led to an idea of using IL-2 in the treatment of those diseases, where their activity is reduced. However, IL-2 is also a potent activator of both NK cells and cytotoxic T cells, and therefore has to be administered in a dose inadequate to activate them, but sufficient to support the function of Treg cells; in other words, era of low-dose IL-2 treatment was initiated. It has been proven to be effective in the therapy of autoimmune diabetes mellitus, SLE, atherosclerosis and alopecia areata (32). It is now recommended to start a 5-day treatment by IL-2 at a dose of one million i.u. per day and repeat it at two weeks intervals. Initial treatment increases the numbers of nTreg cells in the peripheral blood to twice, and maintenance doses cause nTreg cells numbers to be between $20 \%$ and $60 \%$ above baseline. The number of nTreg cells increases not only in peripheral blood, but also in the place of ongoing autoimmune processes (proven in alopecia areata biopsy specimens) (32).

An improved method of afore mentioned therapy is the administration of IL-2 simultaneously with modified human anti-IL-2 monoclonal antibodies $\left(\mathrm{mAb}_{\mathrm{s}}\right)$. These stabilize human IL-2 in a conformation that promotes nTreg cells expansion. The "mAb - 
Tab. 1. Cytokine produced by particular subsets of CD4 $4^{+}$cells.

\begin{tabular}{lccccccc}
\hline Cytokine & Th1 & Th2 & Th9 & Th17 & Th22 & Tfh & Treg \\
\hline IFN- $\gamma$ & ++ & - & - & + & - & - & - \\
\hline IL-2 & ++ & - & - & - & - & - & - \\
\hline IL-4 & - & ++ & - & - & - & - & - \\
\hline IL-5 & - & ++ & - & - & - & - & - \\
\hline IL-6 & - & - & - & + & - & - & - \\
\hline IL-13 & - & ++ & - & - & + & - & - \\
\hline IL-9 & - & - & ++ & - & - & - & - \\
\hline IL-10 & + & ++ & ++ & + & - & - & ++ \\
\hline IL-17 & - & - & - & ++ & - & - & - \\
\hline IL-21 & - & - & - & - & - & ++ & - \\
\hline IL-22 & + & - & - & + & ++ & - & - \\
\hline IL-26 & - & - & - & + & - & - & - \\
\hline IL-35 & - & - & & - & - & - & ++ \\
\hline TNF & ++ & + & - & + & + & - & - \\
\hline Lymphotoxin & ++ & - & - & - & - & - & - \\
\hline
\end{tabular}

IFN- $\gamma$ - interferon gamma, IL - interleukin, Th - T helper cells, TNF - tumor necrotising factor, $\mathrm{Tfh}-\mathrm{T}$ follicular helper cells

IL-2" complex induced T1D remission in a NOD-mouse model (NOD - non-obese diabetic), reducing the intensity of pathological processes in experimental allergic encephalitis, and protecting mice in xenogeneic graft-versus-host disease (GvHD) (33); these results represent a promise for human medicine.

Another treatment option is the administration of nTreg cells alone. These are isolated from the peripheral blood of a patient and multiplied in in vitro conditions (activation with beads coated with anti-TCR and anti-CD28 monoclonal antibodies in the presence of IL-2). Usually, there is a 500 to 2,000-fold increase in their numbers; they are then applied to the patient. It has been shown that Treg cells thus produced (induced Treg $_{\mathrm{s}}-\mathrm{i}_{\mathrm{Treg}}$ ) persist in the patient's body for at least one year. The first phase of successful clinical trials was performed in the treatment of T1D (34).

Instead of expanding Treg cells from peripheral blood, this can also be achieved from cryopreserved umbilical blood (35, 36). The authors report a 211-fold proliferation of cells during 18-days cultivation of Treg cells with beads coated with anti-CD3 and anti-CD28 monoclonal antibodies (mAbs) in the presence of recombinant human IL-2. An even greater yield was obtained, when the umbilical nTreg cells were cultured in vitro with artificial APCs (aAPC) in the presence of anti-CD3 $\mathrm{mAb}_{\mathrm{s}}$ and IL-2. aAPCs represent cells of the K562 tumor line, which were transfected by FCGR1 (coding for a high affinity IgG receptor; CD64) and CD86 genes (CD86 is a ligand for CD28). When umbilical nTreg cells were co-cultivated with $\mathrm{AAPC}_{\mathrm{s}}$, anti-CD3 monoclonal antibodies and IL-2, nTreg cells underwent activation by anti-CD3 mAb previously bound to FCGR1 and by their CD28-molecules upon binding to $\mathrm{CD} 86$ in $\mathrm{aAPC}_{\mathrm{s}}$ membranes; in turn, IL-2 will ensure their proliferation (Fig. 1). The result is a large, up to 13,000-fold, expansion of Treg lymphocytes! Subsequent administration of such expanded iTreg cells significantly reduced the incidence of acute GvH-response $(35,37)$.

The treatment of autoimmune diseases by induced Treg cell has, however, one major disadvantage. This is because iTreg $_{\mathrm{s}}$ are polyspecific, which means, that they can inhibit desired immune processes, when administered to a patient. The starting point is therefore to create such iTreg $\mathrm{s}_{\mathrm{s}}$ that are specific for a given autoantigen only. Here, molecular engineering in the form of creating Treg lymphocytes with a chimeric antigen receptor (CAR) comes to aid.

The formation of cells with an artificial T lymphocyte antigen receptor dates back to the mid-1980s in order to fight tumors more effectively. It is only now that the attention is drawn to the use of CAR technology for other areas. CARs are artificial receptors that allow $\mathrm{T}$ lymphocyte to activate themselves as soon as a specific antigen is detected and not wait for another costimulatory or polarization signals.

CAR is a synthetic structure. At the gene level, the variable genes for the heavy $\left(\mathrm{V}_{\mathrm{H}}\right)$ and light chain $\left(\mathrm{V}_{\mathrm{L}}\right)$ of a monoclonal antibody ( $\mathrm{scFv}$ - single chain variable fragment) specific for an autoantigen are linked to the hinge region exon, usually IgG1-type and the transmembrane region exon, which may be derived from a plurality of transmembrane molecules, such as CD4, CD8 or CD28. These genes will be subsequently linked to genes for the cytoplasmic portion of the future receptor that are responsible for $\mathrm{T}$ cell activation. There are several variations of these cytoplasmic genes. The first generation of CARs consisted of only one exon encoding the CD3 dzéta chain-activating region, the second generation of CARs additionally exon determining the costimulatory molecule CD28, or another co-stimulatory molecule (4-1BB, OX40, DAP10) or both (the third generation CAR-lymphocytes) (38) (Fig. 2).

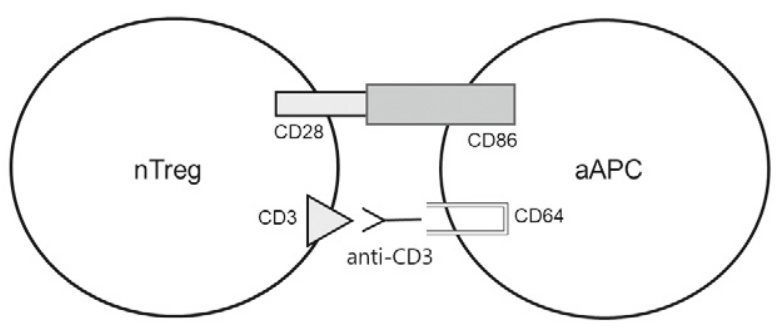

Fig. 1. Activation of nTreg cells by artificial antigen presenting cells (for explanation, see the text)

aAPC - artificial antigen presenting cells.

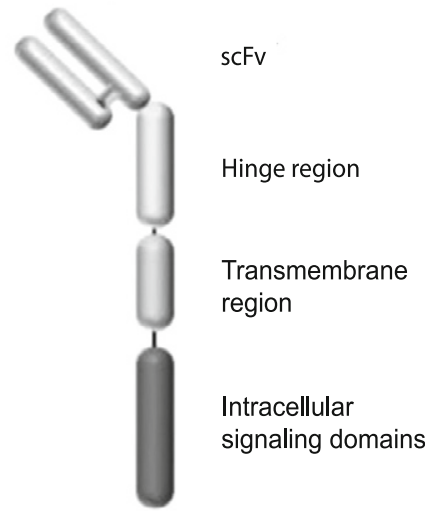

Fig. 2. Structure of chimeric receptor (CAR) (for detailed explanation, see the text.) 
The meaning of existence of antibody variable domains in the construct is not only to bind the given autoantigen, but above all to avoid the phenomenon of immune restriction that would be true if the construct were $\mathrm{scFv}$-domains of $\mathrm{T}$ cell receptor. Indeed, the CAR receptors thus formed can be used to treat multiple patients regardless of their HLA molecules. The CD3 chain-activating region fulfils the same role as in the TCR, i.e. it is the region, (ITAMs) to which tyrosine kinases responsible for activation processes bind. Based on a two signal model of $\mathrm{T}$ cell activation, not only antigen recognition is sufficient, but also signaling, that occurs in costimulatory interactions, typically between CD80 and CD28, must occur as well. And this is what the second and the third cytoplasmic regions of the CAR construct provide (39).

The artificially engineered CAR gene, using retroviral or lentiviral vectors, is transferred to pre-isolated and propagated iTreg cells, which are subsequently transferred to a recipient. CAR lymphocytes specifically recognize autoantigens, activate and promote their immunosuppressive action. This method has been tested in several experimental models with a positive effect (40), which is also promising for use in human medicine in the near future.

Regulatory T cells have been known for more than two decades (7). Regulatory B cells $\left(\right.$ Breg $_{s}$ ) have also recently been added. What is their difference from Treg cells? Above all, it is their origin. While Treg cells are known to be natural and peripheral/induced, in Breg cells we have not yet been able to detect their specific transcription factors, so that they only appear as induced. They arise in an inflammatory environment under the influence of proinflammatory cytokines, molecular recognition patterns (PAMP) or direct autoantigens. Essentially, any B cell, when receives appropriate signals, can differentiate into a Breg cell. Breg cells can perform their immunosuppressive activity by contacting (express FASL, PD1L, CTLA4) and also in a non-contact manner (via IL10 , IL-35, and TGF- $\beta$ synthesis). This ability is also enhanced by the induction of Treg cells by direct co-operation with naive T cells (Th0) involving class II HLA molecules and costimulatory (CD80) molecules (41-43).

Breg cells are a heterogeneous population of cells. They express low IgD as the antigen receptor and different surface markers, including CD5, CD19, CD20, CD21, CD23, CD24, T cell immunoglobulin and mucin domain 1 (TIM-1), and CD138. A marker that is expressed by the majority of reported Breg subsets is CD1d $(41,44)$. Breg cells by presenting glycolipids via their CD1d molecules induce immunosuppressive iNKT cell activity (by induction of IL-10 synthesis). It was shown that Breg cells from SLE patients with active disease expressed decreased levels of CD1d and did not support the expansion and activation of iNKT cells (45). Another defect associated with Breg cells was also observed in SLE. It is characterized among other by an increased IFN- $\alpha$ production by plasmacytoid cells (pDCs) and it was found it had been just Breg cells, whose inadequate function failed to inhibit their over-synthesis of interferons $(46,47)$. This suggests that there is feedback between Breg cells and pDC in healthy subjects, while in SLE patients there is none. Treatment of patients with rituximab (anti-CD20 monoclonal antibodies) resulted in re-emergence of
Breg cells, restoration of NKT-cell function, and improvement of clinical status $(46,47)$.

\section{Reference}

1. Sakaguchi S, Miyara M, Costantino CM, Hafler DA. FOXP3+ regulatory $\mathrm{T}$ cells in the human immune system. Nat Rev Immunol 2010; 10 (7): 490-500.

2. Li X, Zheng Y. Regulatory T cell identity: formation and maintenance. Trends Immunol 2015; 36 (6): 344-353.

3. Van Gool F, Nguyen MLT, Mumbach MR, Satpathy AT, Rosenthal WL, Giacometti S et al. A Mutation in the transcription factor foxp3 drives T helper 2 effector function in regulatory T cells. Immunity 2019; 50 (2): 362-77 e6.

4. Lio CW, Hsieh CS. A two-step process for thymic regulatory T cell development. Immunity 2008; 28 (1): 100-111.

5. Owen DL, Mahmud SA, Sjaastad LE, Williams JB, Spanier JA, Simeonov DR et al. Thymic regulatory T cells arise via two distinct developmental programs. Nat Immunol 2019; 20 (2): 195-205.

6. Bennett CL, Christie J, Ramsdell F, Brunkow ME, Ferguson PJ, Whitesell $\mathbf{L}$ et al. The immune dysregulation, polyendocrinopathy, enteropathy, X-linked syndrome (IPEX) is caused by mutations of FOXP3. Nat Genet 2001; 27 (1): 20-21.

7. Sakaguchi S, Sakaguchi N, Asano M, Itoh M, Toda M. Immunologic self-tolerance maintained by activated $\mathrm{T}$ cells expressing IL-2 receptor alpha-chains (CD25). Breakdown of a single mechanism of selftolerance causes various autoimmune diseases. J Immunol. 1995; 155 (3): 1151-1164.

8. Liu W, Putnam AL, Xu-Yu Z, Szot GL, Lee MR, Zhu S et al. CD127 expression inversely correlates with FoxP3 and suppressive function of human CD4+ T reg cells. J Exp Med 2006; 203 (7): 1701-1711.

9. Akkaya B, Oya Y, Akkaya M, Al Souz J, Holstein AH, Kamenyeva O et al. Regulatory $\mathrm{T}$ cells mediate specific suppression by depleting peptideMHC class II from dendritic cells. Nat Immunol 2019; 20 (2): 218-231.

10. Rissiek A, Baumann I, Cuapio A, Mautner A, Kolster M, Arck PC et al. The expression of $\mathrm{CD} 39$ on regulatory T cells is genetically driven and further upregulated at sites of inflammation. J Autoimmun 2015; 58: 12-20.

11. Shevach EM. Mechanisms of foxp3 + T regulatory cell-mediated suppression. Immunity 2009; 30 (5): 636-645.

12. Liu Z, Gerner MY, Van Panhuys N, Levine AG, Rudensky AY, Germain RN. Immune homeostasis enforced by co-localized effector and regulatory T cells. Nature 2015; 528 (7581): 225-230.

13. Carrizosa E, Mempel TR. Immunology: In the right place at the right time. Nature 2015; 528 (7581): 205-206.

14. Yadav M, Stephan S, Bluestone JA. Peripherally induced tregs - role in immune homeostasis and autoimmunity. Front Immunol 2013; 4: 232.

15. Manzanillo P, Eidenschenk C, Ouyang W. Deciphering the crosstalk among IL-1 and IL-10 family cytokines in intestinal immunity. Trends Immunol 2015; 36 (8): 471-478.

16. Schiering C, Krausgruber T, Chomka A, Frohlich A, Adelmann K, Wohlfert EA et al. The alarmin IL-33 promotes regulatory T-cell function in the intestine. Nature 2014; 513 (7519): 564-568.

17. Feng Y, Arvey A, Chinen T, van der Veeken J, Gasteiger G, Rudensky AY. Control of the inheritance of regulatory T cell identity by a cis element in the Foxp3 locus. Cell 2014; 158 (4): 749-763. 
18. Wing JB, Tanaka A, Sakaguchi S. Human FOXP3 (+) regulatory T cell heterogeneity and function in autoimmunity and cancer. Immunity 2019; 50 (2): 302-316.

19. Gagliani N, Magnani CF, Huber S, Gianolini ME, Pala M, LiconaLimon P et al. Coexpression of CD49b and LAG-3 identifies human and mouse T regulatory type 1 cells. Nat Med 2013; 19 (6): 739-746.

20. Magnani CF, Alberigo G, Bacchetta R, Serafini G, Andreani M, Roncarolo MG et al. Killing of myeloid APCs via HLA class I, CD2 and CD226 defines a novel mechanism of suppression by human Tr1 cells. Eur J Immunol 2011; 41 (6): 1652-1662.

21. Visperas A, Vignali DA. Are regulatory $\mathrm{T}$ cells defective in type 1 diabetes and can we fix them? J Immunol 2016; 197 (10): 3762-3770.

22. Chavele KM, Ehrenstein MR. Regulatory T-cells in systemic lupus erythematosus and rheumatoid arthritis. FEBS Lett 2011; 585 (23): 3603-3610.

23. Li H, Yang D, Tang Z. Bcl2 like protein-12 suppresses Foxp3 (+) regulatory $\mathrm{T}$ cells in patients with rheumatoid arthritis. Am J Transl Res 2019; 11 (5): 3048-3055.

24. Balandina A, Lecart S, Dartevelle P, Saoudi A, Berrih-Aknin S. Functional defect of regulatory CD4 $(+) \mathrm{CD} 25+\mathrm{T}$ cells in the thymus of patients with autoimmune myasthenia gravis. Blood 2005; 105 (2): 735-741.

25. Kumar M, Putzki N, Limmroth V, Remus R, Lindemann M, Knop D et al. CD4+CD25+FoxP3+ T lymphocytes fail to suppress myelin basic protein-induced proliferation in patients with multiple sclerosis. J Neuroimmunol 2006; 180 (1-2): 178-184.

26. Buc M. Role of regulatory $T$ cells in pathogenesis and biological therapy of multiple sclerosis. Mediators Inflamm 2013; 2013: 963748

27. Buckner JH. Mechanisms of impaired regulation by CD4 (+)CD25 $(+)$ FOXP3 $(+)$ regulatory T cells in human autoimmune diseases. Nat Rev Immunol 2010; 10 (12): 849-859.

28. Yamada A, Arakaki R, Saito M, Tsunematsu T, Kudo Y, Ishimaru N. Role of regulatory $\mathrm{T}$ cell in the pathogenesis of inflammatory bowel disease. World J Gastroenterol 2016; 22 (7): 2195-2205.

29. Mao C, Wang S, Xiao Y, Xu J, Jiang Q, Jin M et al. Impairment of regulatory capacity of $\mathrm{CD} 4+\mathrm{CD} 25+$ regulatory $\mathrm{T}$ cells mediated by dendritic cell polarization and hyperthyroidism in Graves' disease. J Immunol 2011; 186 (8): 4734-4743.

30. Boyer O, Saadoun D, Abriol J, Dodille M, Piette JC, Cacoub P et al. $\mathrm{CD} 4+\mathrm{CD} 25+$ regulatory T-cell deficiency in patients with hepatitis C-mixed cryoglobulinemia vasculitis. Blood 2004; 103 (9): 3428-3430.

31. Ohl K, Tenbrock K. Regulatory T cells in systemic lupus erythematosus. Eur J Immunol 2015; 45 (2): 344-355.

32. Klatzmann D, Abbas AK. The promise of low-dose interleukin-2 therapy for autoimmune and inflammatory diseases. Nat Rev Immunol 2015; 15 (5): 283-294.
33. Trotta E, Bessette PH, Silveria SL, Ely LK, Jude KM, Le DT et al. A human anti-IL-2 antibody that potentiates regulatory $\mathrm{T}$ cells by a structure-based mechanism. Nat Med 2018; 24 (7): 1005-1014.

34. Bluestone JA, Buckner JH, Fitch M, Gitelman SE, Gupta S, Hellerstein MK et al. Type 1 diabetes immunotherapy using polyclonal regulatory T cells. Sci Transl Med 2015; 7 (315): 315ra189.

35. Brunstein CG, Miller JS, McKenna DH, Hippen KL, DeFor TE, Sumstad D et al. Umbilical cord blood-derived T regulatory cells to prevent GVHD: kinetics, toxicity profile, and clinical effect. Blood 2016; 127 (8): 1044-1051.

36. Sharma A, Rudra D. Regulatory $\mathrm{T}$ cells as therapeutic targets and mediators. Int Rev Immunol 2019: 1-21.

37. Parmar S, Shpall EJ. Treg adoptive therapy: is more better? Blood 2016; 127 (8): 962-923.

38. Dai H, Wang Y, Lu X, Han W. Chimeric antigen receptors modified T-cells for cancer therapy. J Natl Cancer Inst 2016; 108 (7).

39. Jensen MC, Riddell SR. Design and implementation of adoptive therapy with chimeric antigen receptor-modified T cells. Immunol Rev 2014; 257 (1): 127-144.

40. Wu M, Zhang L, Zhang H, Ning J, Tu S, He Y et al. CD19 chimeric antigen receptor-redirected $\mathrm{T}$ cells combined with epidermal growth factor receptor pathway substrate 8 peptide-derived dendritic cell vaccine in leukemia. Cytotherapy 2019; 21 (6): 659-670.

41. Rosser EC, Mauri C. Regulatory B cells: origin, phenotype, and function. Immunity 2015; 42 (4): 607-612.

42. Ray A, Khalil MI, Pulakanti KL, Burns RT, Gurski CJ, Basu S et al. Mature $\operatorname{IgD}(\mathrm{low} /-) \mathrm{B}$ cells maintain tolerance by promoting regulatory T cell homeostasis. Nat Commun 2019; 10 (1): 190.

43. Yang M, Rui K, Wang S, Lu L. Regulatory B cells in autoimmune diseases. Cell Mol Immunol 2013; 10 (2): 122-132.

44. Maravillas-Montero JL, Acevedo-Ochoa E. Human B regulatory cells: the new players in autoimmune disease. Rev Invest Clin 2017; 69 (5): 243-26.

45. Oleinika K, Rosser EC, Matei DE, Nistala K, Bosma A, Drozdov I et al. CD1d-dependent immune suppression mediated by regulatory B cells through modulations of iNKT cells. Nat Commun 2018; 9 (1): 684.

46. Mauri C, Menon M. Human regulatory B cells in health and disease: therapeutic potential. J Clin Invest 2017; 127 (3): 772-779.

47. Menon M, Blair PA, Isenberg DA, Mauri C. A regulatory feedback between plasmacytoid dendritic cells and regulatory B cells is aberrant in systemic lupus erythematosus. Immunity 2016; 44 (3): 683-697.

Received July 23, 2019. Accepted Septe,ber 25, 2019. 